\section{Genetic library construction and screening}

\author{
Genetic Library Construction and Screening \\ RC Bird and BF Smith \\ Springer-Verlag, Heidelberg; 2002. 241 pp. \\ $€ 79.95$, ring binder. ISBN 3-540-67278-8
}

Heredity (2003) 91, 539. doi:10.1038/sj.hdy.6800364

Reviewed by H Isaacs

This somewhat cryptically titled offering in the Springer Lab Manual series has the stated intention of exposing scientists to a set of techniques that have rapidly become 'core' technologies for those of us interested in gene expression. The emphasis is very much on cDNA, and I think the book could just as easily have been entitled "cDNA Library Construction and Screening", which is not to say that those of a more genomic persuasion would not find something of interest here.

Each of the chapters in this multi-author volume has a reasonably consistent and accessible format. There is an introduction/overview, usually containing an appropriate flow chart for the technique under discussion. Extensive lists of the required materials are included, and the procedures are laid out in a step-by-step numbered format that I found generally pretty easy to follow. A trouble-shooting guide is also usually included. Finally, a short reference list is provided, along with a very useful set of contact details for relevant suppliers.

The book itself is divided into three sections. The first section is something of an introduction, and Chapter 1 includes lots of homely advice on the basics of cDNA library construction. To be honest, it is a bit short on detail, but still potentially useful to those new to the field. Other topics covered in this section include cloning of PCR products and the use of PCR in site-directed mutagenesis and construction of cDNA libraries from small quantities of mRNA. I must admit I had some issues with the chapter on cloning of PCR products. The discussion was not very broad, and one of the most commonly used strategies for cloning PCR products, that is, including the restriction sites in the primers, is barely mentioned at all. The second section contains extensive chapters on subtractive hybridization and differential display. These two techniques, once the centrepiece of many grant proposals and bane of countless graduate students' lives, are described in a fair amount of detail. However, with the advance of genome and EST projects, combined with genome-scale DNA arrays, the use of these techniques for analysing differential gene expression may become somewhat limited. The final section cherry-picks a few noteworthy topics, including yeast one- and two-hybrid cloning, peptide-presenting phage libraries and high throughput screening of membranegridded libraries. If you have a requirement for doing one of these procedures, the chapters provide a flavour for the technique and may provide enough information to get the experienced investigator going.

So, who is this book aimed at? The editors state that graduate students and more senior scientists should be able to get something from their book. I think I probably agree with them. The book is really an odd mixture, containing very basic stuff, like cloning of PCR products, but also advanced topics like yeast two-hybrid systems and differential display. I can certainly envisage making use of it again, and I won't be afraid to point a graduate student to an appropriate chapter. If you want advice on the strategy that you need to adopt in order to answer a particular problem, this isn't the book for you. But could this book get you up and running with a particular technique? The answer is probably "yes", if you were reasonably experienced in general molecular biology. You can't ask for more than that. I have one gripe with Springer and that is the typos. I did wonder what was on offer in Chapter 1, "Strategies for cDNA Cling".

H Isaacs

Department of Biology, University of York, Area 11, York YO10 5YW,UK E-mail: hvi1@york.ac.uk 